\title{
The Berkani's property and a note on some recent results
}

\author{
Zakariae Aznay, Hassan Zariouh
}

\begin{abstract}
In this paper, we continue the study of property $\left(U W_{\Pi}\right)$ introduced in $[5$, in connection with other Weyl type theorems. Moreover, we give counterexamples to show that some recent results related to this property, which are announced and proved by P. Aiena and M. Kachad in [2] are false. Furthermore, we specify the mistakes committed in each of them and we give the correct versions. We also give a global note on the paper [7].
\end{abstract}

\section{Introduction}

We continue the study of properties introduced in [4, 5, in connection with other properties and Weyl type theorems. Moreover, we prove by counterexample (see Remark I), that we do not expected that an operator satisfying property $\left(U W_{\Pi}\right)$ satisfies property $\left(Z_{\Pi_{a}}\right)$, contrarily to what has been proved in [2, Thorem 2.5]. Furthermore, we give the correct version of 2, Thorem $2.5]$ by proving in Theorem [3.7, that if $T \in L(X)$ is an operator satisfying property $\left(U W_{\Pi}\right)$ and $\Pi_{a}(T) \cap \sigma_{u w}(T)=\emptyset$, then it satisfies property $\left(Z_{\Pi_{a}}\right)$. We also give the correct version of [2, Thorem 2.6] (see Remark II and Theorem 3.12). As deduction, we give analogous result to Theorem 3.12 for the properties $\left(U W_{E}\right)$ and $\left(Z_{E_{a}}\right)$. And, we give in Remark IV a very crucial observation on the results of the paper [7].

\section{Terminology and preliminaries}

Let $X$ denote an infinite dimensional complex Banach space, and we denote by $L(X)$ the algebra of all bounded linear operators on $X$. For $T \in L(X)$, we denote by $\alpha(T)$ the dimension of the kernel $\mathcal{N}(T)$ and by $\beta(T)$ the codimension of the range $\mathcal{R}(T)$. By $\sigma(T)$ and $\sigma_{a}(T)$, we denote the spectrum, the approximate spectrum of $T$, respectively. For an operator $T \in L(X)$, the ascent $p(T)$ and the descent $q(T)$ of $T$ are defined by $p(T)=\inf \left\{n \in \mathbb{N}: \mathcal{N}\left(T^{n}\right)=\mathcal{N}\left(T^{n+1}\right)\right\}$ and $q(T)=\inf \left\{n \in \mathbb{N}: \mathcal{R}\left(T^{n}\right)=\mathcal{R}\left(T^{n+1}\right)\right\}$, respectively; the infimum over the empty set is taken $\infty$. In order to simplify, and to give a global view to the reader, we use the same symbols and notations used in [2]. For more details on the several classes and spectra originating from Fredholm theory and B-Fredholm theory, we refer the reader to [2, 5, 8.

\footnotetext{
${ }^{0} 2010$ AMS subject classification: Primary 47A53, 47A10, 47A11 Keywords: Property $\left(U W_{\Pi}\right)$, Property $\left(Z_{\Pi_{a}}\right)$
} 
In the following list, we summarize the same notations and symbols used in [2], which will be needed in this paper.

The symbol $\bigsqcup$ stands for disjoint union, iso $A$ : isolated points of a subset $A \subset \mathbb{C}$, $A^{C}$ : the complementary of a subset $A \subset \mathbb{C}$.

$\sigma_{b}(T)$ : Browder spectrum of $T$ $\sigma_{u b}(T)$ : upper semi-Browder spectrum of $T$ $\sigma_{w}(T)$ : Weyl spectrum of $T$

$\sigma_{b w}(T)$ : B-Weyl spectrum of $T$

$\sigma_{u w}(T)$ : upper semi-Weyl spectrum of $T$

$\sigma_{u b w}(T)$ : upper semi-B-Weyl spectrum of $T$

$\sigma_{l d}(T)$ : left Drazin spectrum of $T$

$\sigma_{d}(T)$ : Drazin spectrum of $T$,

$\sigma_{p}(T)$ : eigenvalues of $T$

$\sigma_{p}^{0}(T)$ : eigenvalues of $T$ of finite multiplicity

$\Delta_{a}(T):=\sigma_{a}(T) \backslash \sigma_{u w}(T)$

$$
\begin{aligned}
& \Delta^{g}(T):=\sigma(T) \backslash \sigma_{b w}(T) \\
& \Delta_{a}^{g}(T):=\sigma_{a}(T) \backslash \sigma_{u b w}(T) \\
& \Delta(T):=\sigma(T) \backslash \sigma_{w}(T) \\
& \Pi^{0}(T): \text { poles of } T \text { of finite rank } \\
& \Pi_{a}(T): \text { left poles of } T \\
& \Pi_{a}^{0}(T): \text { left poles of } T \text { of finite rank } \\
& \Pi(T): \text { poles of } T \\
& E_{a}(T):=\text { iso } \sigma_{a}(T) \cap \sigma_{p}(T) \\
& E(T):=\text { iso } \sigma(T) \cap \sigma_{p}(T) \\
& E^{0}(T):=\text { iso } \sigma(T) \cap \sigma_{p}^{0}(T) \\
& E_{a}^{0}(T):=\text { iso } \sigma_{a}(T) \cap \sigma_{p}^{0}(T)
\end{aligned}
$$

The following property has relevant role in local spectral theory: a bounded linear operator $T \in L(X)$ is said to have the single-valued extension property (SVEP for short) at $\lambda \in \mathbb{C}$ if for every open neighborhood $U_{\lambda}$ of $\lambda$, the function $f \equiv 0$ is the only analytic solution of the equation

$(T-\mu I) f(\mu)=0 \quad \forall \mu \in U_{\lambda}$. We denote by $\mathcal{S}(T)=\{\lambda \in \mathbb{C}: T$ does not have the SVEP at $\lambda\}$ and we say that $T$ has SVEP if $\mathcal{S}(T)=\emptyset$. We say that $T$ has the SVEP on $A \subset \mathbb{C}$, if $T$ has the SVEP at every $\lambda \in A$. (For more details about this property, we refer the reader to [1]). Thus it follows easily that $T \in L(X)$ has the SVEP at every point of the boundary $\partial \sigma(T)$ of the spectrum $\sigma(T)$. In particular, $T$ has the SVEP at every isolated point in $\sigma(T)$. We also have from [1, Theorem $2.65]$,

$$
p\left(T-\lambda_{0} I\right)<\infty \Longrightarrow \text { T has the SVEP at } \lambda_{0}, \quad(A)
$$

and dually

$$
q\left(T-\lambda_{0} I\right)<\infty \Longrightarrow T^{*} \text { has the SVEP at } \lambda_{0},(B),
$$

Furthermore, if $T-\lambda_{0} I$ is semi-B-Fredholm then the implications above are equivalences.

Note that it well known that if $T^{*}$ has the SVEP on $\left(\sigma_{u b w}(T)\right)^{C}$, then $\sigma_{a}(T)=\sigma(T), \sigma_{w}(T)=$ $\sigma_{u w}(T), \sigma_{b w}(T)=\sigma_{u b w}(T), E(T)=E_{a}(T)$ and $\Pi(T)=\Pi_{a}(T)$. And this gives a short and simple proof of [3, Theorem 2.15].

\section{Correct versions and addendums}

Definition 3.1. 4, 5] Let $T \in L(X) . T$ is said to satisfy

a) property $\left(U W_{\Pi}\right)$ if $\Delta_{a}(T)=\Pi(T)$.

b) property $\left(U W_{E}\right)$ if $\Delta_{a}(T)=E(T)$.

c) property $\left(U W_{E_{a}}\right)$ if $\Delta_{a}(T)=E_{a}(T)$.

We begin this section by the following lemma, which will be useful in the sequel. 
Lemma 3.2. let $T \in L(X)$.

(i) If $T$ satisfies property $\left(U W_{E_{a}}\right)$, then

$E_{a}(T)=E_{a}^{0}(T)=\Pi_{a}^{0}(T)=\Pi_{a}(T)$ and $E(T)=E^{0}(T)=\Pi^{0}(T)=\Pi(T)$.

(ii) If $T$ satisfies property $\left(U W_{E}\right)$, then $E(T)=E^{0}(T)=\Pi_{a}^{0}(T)=\Pi^{0}(T)=\Pi(T)$.

(iii) If $T$ satisfies property $\left(U W_{\Pi}\right)$, then $\Pi^{0}(T)=\Pi(T)=\Pi_{a}^{0}(T)$.

Proof. (i) See, 4, Remark 3.4, Theorem 3.5, Remark 2.5].

(ii) Since $T$ satisfies property $\left(U W_{E}\right)$, then $\lambda \in E(T) \Longleftrightarrow \lambda \in$ iso $\sigma_{a}(T) \cap\left(\sigma_{u w}(T)\right)^{C} \Longleftrightarrow \lambda \in$ $\Pi_{a}^{0}(T)$. So $E(T)=E^{0}(T)=\Pi_{a}^{0}(T)$ and $\Pi^{0}(T)=\Pi(T)$. We show $E^{0}(T)=\Pi^{0}(T)$ and let $\lambda \in E^{0}(T)$ be arbitrary. Then $T$ and $T^{*}$ have the SVEP at $\lambda$. As $\lambda \in$ iso $\sigma(T) \cap\left(\sigma_{u w}(T)\right)^{C}$. Hence $\lambda \in \Pi^{0}(T)$ and consequently, $E^{0}(T)=\Pi^{0}(T)$. As conclusion, we have $E(T)=E^{0}(T)=\Pi_{a}^{0}(T)=\Pi^{0}(T)=$ $\Pi(T)$.

(iii) Goes similarly with (ii).

From Lemma 3.2, we obtain immediately the following corollary.

Corollary 3.3. Let $T \in L(X)$. Then $T$ satisfies property $\left(U W_{E}\right)$ if and only if $T$ satisfies property $\left(U W_{\Pi}\right)$ and $E(T)=\Pi(T)$.

By Corollary 3.3, if $T$ satisfies property $\left(U W_{E}\right)$, then it satisfies property $\left(U W_{\Pi}\right)$. But the converse is generally not true, as we can see in the following example.

Example 3.4. Hereafter, the Hilbert space $l^{2}(\mathbb{N})$ is denoted by $l^{2}$. We consider the operator $T$ defined on $l^{2}$ by $T\left(x_{1}, x_{2}, x_{3}, \ldots\right)=\left(\frac{x_{2}}{2}, \frac{x_{3}}{3}, \frac{x_{4}}{4}, \ldots\right)$. It easily seen that $\sigma_{a}(T)=\sigma_{u w}(T)=\{0\}=$ $E(T)$. Moreover, $\Pi(T)=\emptyset$, since $p(T)=\infty$. So $T$ satisfies property $\left(U W_{\Pi}\right)$, but it does not satisfy property $\left(U W_{E}\right)$. Note here that $\Pi(T) \neq E(T)$.

\section{Remark 3.5.}

1. The properties $\left(U W_{E_{a}}\right)$ and $\left(U W_{\Pi}\right)$ are independent. We consider the operator $T$ defined on the Banach space $l^{2} \oplus l^{2}$ by $T=R \oplus P$, where $R$ is the right shift operator defined on $l^{2}$ by $R\left(x_{1}, x_{2}, x_{3}, \ldots\right)=\left(0, x_{1}, x_{2}, x_{3}, \ldots\right)$ and $P$ is the operator defined on $l^{2}$ by $P\left(x_{1}, x_{2}, x_{3}, \ldots\right)=\left(0, x_{2}, x_{3}, x_{4}, \ldots\right)$. Then $\sigma_{a}(T)=C(0,1) \cup\{0\}, \sigma_{u w}(T)=C(0,1) ;$ where $C(0,1)$ is the unit circle of $\mathbb{C}, E_{a}(T)=\{0\}$ and $\Pi(T)=E(T)=\emptyset$. Thus $T$ satisfies property $\left(U W_{E_{a}}\right)$, but it does not satisfy property $\left(U W_{\Pi}\right)$ and hence it does not satisfy property $\left(U W_{E}\right)$ too. On the other hand, the operator $T$ defined on $l^{2} \oplus l^{2}$ by $T=R \oplus 0$ satisfies property $\left(U W_{\Pi}\right)$, but it does not satisfy property $\left(U W_{E_{a}}\right)$. Indeed $\sigma_{a}(T)=\sigma_{u w}(T)=C(0,1) \cup\{0\}$, $\Pi(T)=E(T)=\emptyset$ and $E_{a}(T)=\{0\}$.

2. The same examples defined above in this remark, show that the properties $\left(U W_{E_{a}}\right)$ and $\left(U W_{E}\right)$ are independent (see also [5]).

Now we prove in Remark I, Remark II and Remark III bellow, by counterexamples that some results announced and proved by P. Aiena, M. Kachad in [2] are false. Moreover, we specify the mistakes committed in each of them and we give there correct versions. 
We recall that a bounded linear operator $T \in L(X)$ is said to satisfy property $\left(Z_{\Pi_{a}}\right)$ if $\Delta(T)=$ $\Pi_{a}(T)$, see [8].

Remark I: It is proved in [2, Theorem 2.5] that if $T \in L(X)$ satisfies property $\left(U W_{\Pi}\right)$, then it satisfies property $\left(Z_{\Pi_{a}}\right)$. But this result is false. Indeed, if we consider the operator $T=R \oplus 0$ defined above in Remark [3.5] then $\sigma(T)=\sigma_{w}(T)=D(0,1)$; where $D(0,1)$ is the closed unit disc in $\mathbb{C}$. Furthermore, it is easily seen that $\sigma_{u b w}(T)=\sigma_{l d}(T)=C(0,1)$ and so $\Pi_{a}(T)=\{0\}$. Hence $T$ satisfies property $\left(U W_{\Pi}\right)$, since $\Delta_{a}(T)=\emptyset=\Pi(T)$, but it does not satisfy property $\left(Z_{\Pi_{a}}\right)$, since $\Delta(T)=\emptyset \neq \Pi_{a}(T)$.

The mistake made in the proof of [2, Theorem 2.5] is as follows: the equality

$$
\sigma(T)=\left(\sigma_{w}(T) \backslash \sigma_{u w}(T)\right) \bigsqcup \Pi_{a}(T) \bigsqcup \sigma_{u b w}(T)
$$

proved in line 8 of the proof of [2, Theorem 2.5] gave the following incorrect decision $\left(I_{1}\right)$ (see line 9 and 10 of the proof of [2, Theorem 2.5]):

Since $\sigma_{u b w}(T) \subset \sigma_{u w}(T)$ then

$$
\sigma(T) \subset\left(\sigma_{w}(T) \backslash \sigma_{u w}(T)\right) \bigsqcup \Pi_{a}(T) \bigsqcup \sigma_{u w}(T)=\sigma_{w}(T) \bigsqcup \Pi_{a}(T) . \quad\left(I_{1}\right)
$$

And the inclusion $\left(I_{1}\right)$ gave the incorrect equality $\left(I_{2}\right)$ bellow (see line 11 of the proof of [2, Theorem 2.5])

$$
\sigma(T)=\sigma_{w}(T) \bigsqcup \Pi_{a}(T) . \quad\left(I_{2}\right)
$$

But here we don't have always the following disjoint union $\left(\sigma_{w}(T) \backslash \sigma_{u w}(T)\right) \bigsqcup \Pi_{a}(T) \bigsqcup \sigma_{u w}(T)$ [even if $T$ satisfies property $\left(U W_{\Pi}\right)$ ], since $\Pi_{a}(T) \cap \sigma_{u w}(T)$ is not necessarily empty. Indeed, It already mentioned that the operator $T=R \oplus 0$ defined above satisfies property $\left(U W_{\Pi}\right)$. But since $\sigma_{u w}(T)=C(0,1) \cup\{0\}$ and $\Pi_{a}(T)=\{0\}$, then $\sigma_{u w}(T) \cap \Pi_{a}(T)=\{0\} \neq \emptyset$. Moreover, this example shows that $\sigma_{w}(T) \cap \Pi_{a}(T)=\{0\} \neq \emptyset$. Thus we cannot consider the union $\sigma_{w}(T) \cup \Pi_{a}(T)$ as a disjoint union considered in the second member of the inclusion $\left(I_{1}\right)$. Consequently, $\sigma_{w}(T) \cap$ $\Pi_{a}(T) \neq \emptyset$ contrarily to what has been proved in the equality $\left(I_{2}\right)$ above. Note that here we have $\sigma(T) \backslash \sigma_{w}(T)=\emptyset \neq \Pi_{a}(T)=\{0\}$.

We recall that if we have two sets $A$ and $B$ of $\mathbb{C}$ such that there union is disjoint and $B$ is a subset of $C$, then we cannot expected generally that the union $A \cup C$ will be disjoint. And this was exactly the origin of the error made in the inclusion $\left(I_{1}\right)$.

Among other results, we prove in the next proposition that the properties $\left(U W_{\Pi}\right)$ and $\left(Z_{\Pi_{a}}\right)$ are independent.

Proposition 3.6. The properties $\left(U W_{\Pi}\right)$ and $\left(Z_{\Pi_{a}}\right)$ are independent.

Proof. It already mentioned in Remark I, that the operator $T=R \oplus 0$ satisfies property $\left(U W_{\Pi}\right)$, but it does not satisfy property $\left(Z_{\Pi_{a}}\right)$. On the other hand, if we consider the operator $T=R \oplus R \oplus L$; where $L$ is the left shift operator. Then $\sigma(T)=\sigma_{a}(T)=\sigma_{w}(T)=D(0,1)$ and so $\Delta(T)=\Pi(T)=$ $\Pi_{a}(T)=\emptyset$. Thus $T$ satisfies property $\left(Z_{\Pi_{a}}\right)$. Moreover, we have $\alpha(T)=1$ and $\beta(T)=2$, then $0 \in \Delta_{a}(T)$. Hence $T$ does not satisfy property $\left(U W_{\Pi}\right)$. 
We give in the following theorem the correct versions of [2, Theorem 2.5].

Theorem 3.7. Let $T \in L(X)$ be an operator satisfying property $\left(U W_{\Pi}\right)$. Then If $\Pi_{a}(T) \cap \sigma_{u w}(T)=\emptyset$, then $T$ satisfies property $\left(Z_{\Pi_{a}}\right)$. In particular, property $\left(Z_{\Pi_{a}}\right)$ holds for $T$ if $\sigma_{u w}(T)=\sigma_{u b w}(T)$.

Proof. It is clear that $\Delta(T) \subset \Delta_{a}(T)$. By hypothesis, $\Pi_{a}(T) \cap \sigma_{u w}(T)=\emptyset$, it follows that $\Pi_{a}(T)=$ $\Pi_{a}(T) \cap\left(\sigma_{u w}(T)\right)^{C} \subset \Delta_{a}(T)$. As $T$ satisfies property $\left(U W_{\Pi}\right)$ then from Lemma 3.2, $\Delta_{a}(T)=$ $\Pi^{0}(T)$. The inclusion $\Pi^{0}(T) \subset \Delta(T)$ is always true. Hence $\Delta(T)=\Pi_{a}(T)$ and $T$ satisfies $\left(Z_{\Pi_{a}}\right)$.

In particular, if $\sigma_{u w}(T)=\sigma_{u b w}(T)$, then $\Pi_{a}(T) \cap \sigma_{u w}(T)=\Pi_{a}(T) \cap \sigma_{u b w}(T)=\emptyset$. So property $\left(Z_{\Pi_{a}}\right)$ holds for $T$.

Following [8], we say that an operator $T \in L(X)$ satisfies property $\left(Z_{E_{a}}\right)$ if $\Delta(T)=E_{a}(T)$. Similarly to Theorem 3.7, and to avoid redundancies, we give the following result without proof. Note that the examples given in Proposition 3.6 shows that the properties $\left(U W_{E}\right)$ and $\left(Z_{E_{a}}\right)$ are independent.

Theorem 3.8. Let $T \in L(X)$. The following statement holds.

If $T$ satisfies property $\left(U W_{E}\right)$ and $E_{a}(T) \cap \sigma_{u w}(T)=\emptyset$, then $T$ satisfies property $\left(Z_{E_{a}}\right)$.

In the first assertion of the next theorem, we extend with simple and short proof [3, Theorem $2.15]$ in which it is proved that if $T^{*}$ has the SVEP, then the properties $\left(U W_{\Pi}\right)$ and $\left(Z_{\Pi_{a}}\right)$ for $T$ are equivalent. Note that property $(g b)$ is equivalent to say that $T^{*}$ has the SVEP on $\Delta_{a}^{g}(T)$. Recall that according to [6], an operator $T \in L(X)$ is said to satisfy property $(g b)$ if $\Delta_{a}^{g}(T)=\Pi(T)$.

Theorem 3.9. Let $T \in L(X)$. We have the following statements.

(i) If $T$ satisfies property $(g b)$, then the properties $\left(U W_{\Pi}\right)$ and $\left(Z_{\Pi_{a}}\right)$ for $T$ are equivalent.

(ii) If $T$ satisfies the properties $\left(U W_{\Pi}\right)$ and $\left(Z_{\Pi_{a}}\right)$, then $T$ satisfies property $(g b)$.

(iii) If $T \in L(X)$ satisfies the property $\left(Z_{\Pi_{a}}\right)$, then $\Pi(T) \subset \Delta_{a}(T)$.

Proof. (i) Suppose that $T$ satisfies property $\left(U W_{\Pi}\right)$. The property $(g b)$ for $T$ entails from 6 , Corollary 2.9] and Lemma 3.2 that $\Delta(T)=\Pi^{0}(T)=\Pi(T)=\Pi_{a}(T)$. So $T$ satisfies property $\left(Z_{\Pi_{a}}\right)$. Conversely, if $T$ satisfies property $\left(Z_{\Pi_{a}}\right)$, then from [6, Theorem 2.3] and [8, Lemma 2.9] that $\Delta_{a}(T)=\Pi^{0}(T)=\Pi(T)$.

(ii) The properties $\left(U W_{\Pi}\right)$ and $\left(Z_{\Pi_{a}}\right)$ imply from [8, Lemma 2.9], that $\Delta_{a}(T)=\Pi^{0}(T)$ and $\Pi(T)=\Pi_{a}(T)$. This is equivalent from [6, Theorem 2.10] to say that $T$ satisfies property $(g b)$.

(iii) Since $T$ satisfies the property $\left(Z_{\Pi_{a}}\right)$, then $\Pi(T)=\Pi_{a}(T)=\Delta(T)$. As $\Delta(T) \subset \Delta_{a}(T)$, it follows that $\Pi(T) \subset \Delta_{a}(T)$.

\section{Remark 3.10.}

1. Remark that all of the properties $(g b),\left(U W_{\Pi}\right)$ and $\left(Z_{\Pi_{a}}\right)$ are mutually independent.

i. We consider the operator $T$ defined on $l^{2}$ by $T\left(x_{1}, x_{2}, x_{3}, \ldots\right)=\left(x_{2}, 0,0, \ldots\right)$. Then $\sigma(T)=$ $\sigma_{a}(T)=\sigma_{w}(T)=\sigma_{u w}(T)=\Pi_{a}(T)=\Pi(T)=\{0\}$ and $\sigma_{u b w}(T)=\emptyset$. So $T$ satisfies property $(g b)$, but it does not satisfy neither property $\left(U W_{\Pi}\right)$ nor property $\left(Z_{\Pi_{a}}\right)$. 
ii. The operator $T=R \oplus R \oplus L$ defined in Proposition 3.6 satisfies property $\left(Z_{\Pi_{a}}\right)$, but it does not satisfy property $(g b)$, since $0 \in \Delta_{a}^{g}(T)$.

iii. The operator $T=R \oplus 0$ defined above in Remark 3.5 satisfies property $\left(U W_{\Pi}\right)$, but it does not satisfy property $(g b)$.

2. Remark that all of the properties $(g b),\left(U W_{E}\right),\left(U W_{E_{a}}\right)$ and $\left(Z_{\Pi_{a}}\right)$ are mutually independent.

i. We consider the operator $T$ defined on $l^{2}$ by $T\left(x_{1}, x_{2}, x_{3}, \ldots\right)=\left(x_{2}, 0,0, \ldots\right)$. Then $T$ satisfies property $(g b)$, but it does not satisfy neither property $\left(U W_{E}\right)$ nor property $\left(U W_{E_{a}}\right)$.

ii. The operator $T=R \oplus 0$ defined above satisfies property $\left(U W_{E}\right)$, but it does not satisfy neither property $(g b)$ nor property $\left(Z_{\Pi_{a}}\right)$.

iii. The operator $T=R \oplus R \oplus L$ defined above satisfies property $\left(Z_{\Pi_{a}}\right)$, but it does not satisfy neither property $\left(U W_{E}\right)$ nor property $\left(U W_{E_{a}}\right)$. And the operator $T=R \oplus P$ defined in Remark 3.5. satisfies property $\left(U W_{E_{a}}\right)$, but it does not satisfy neither property $(g b)$ nor property $\left(Z_{\Pi_{a}}\right)$.

Remark II: P. Aiena and M. Kachad announced and proved in [2, Theorem 2.6] the following result.

Theorem: 2, Theorem 2.6] Let $T \in L(X)$. Then the following statements are equivalent:

(i) $T$ satisfies property $\left(U W_{\Pi}\right)$;

(ii) $T$ satisfies property $\left(Z_{\Pi_{a}}\right)$ and $\sigma_{w}(T) \backslash \sigma_{u w}(T)=\sigma(T) \backslash \sigma_{a}(T)$;

(iii) $T$ satisfies property $\left(Z_{\Pi_{a}}\right)$ and $\Delta_{a}(T) \cap \sigma_{w}(T)=\emptyset$.

But this theorem is not true. Indeed, by Proposition 3.6 it follows that the implications "(i) $\Longrightarrow($ ii $)$ " and " $(i) \Longrightarrow($ iii $)$ " are false. Note that the reasoning they used for proving [2, Theorem 2.6] is as follows: they proved that "(i) $\Longleftrightarrow(i i)$ " and "(i) $\Longleftrightarrow($ iii $)$ " and they deduced by transitivity that "(ii) $\Longleftrightarrow($ iii $)$ ". But, since the properties $\left(U W_{\Pi}\right)$ and $\left(Z_{\Pi_{a}}\right)$ are independent (see Proposition 3.6), then the proof of "(ii) $\Longleftrightarrow$ (iii)" remains incorrect.

Moreover, in line 12 and 13 and 14 of the proof of [2, Theorem 2.6], they said

"If $\lambda \in \Delta_{a}(T)$ then $\lambda \notin \sigma_{w}(T)$ and hence $\lambda \notin \sigma_{u w}(T)$. This implies that $\lambda \in \Delta_{a}(T)=\Pi_{a}(T)=\Pi(T)$ "

But this is not clear.

Now, we give in Theorem 3.12 bellow, the correct version of [2, Theorem 2.6] in which we also prove that the equivalence "(ii) $\Longleftrightarrow($ iii $)$ " of [2, Theorem 2.6] is true. Before that, we give the following lemma.

Lemma 3.11. The following equivalence holds for every $T \in L(X)$.

$$
\Delta_{a}(T) \cap \sigma_{w}(T)=\emptyset \Longleftrightarrow \sigma_{w}(T) \backslash \sigma_{u w}(T)=\sigma(T) \backslash \sigma_{a}(T) .
$$


Proof. $\Longrightarrow)$ Suppose that $\Delta_{a}(T) \cap \sigma_{w}(T)=\emptyset$, then $\left(\sigma_{w}(T) \backslash \sigma_{u w}(T)\right) \cap \sigma_{a}(T)=\emptyset$. If $\lambda \in \sigma_{w}(T) \backslash \sigma_{u w}(T)$, then $\lambda \notin \sigma_{a}(T)$, so that $\lambda \in \sigma(T) \backslash \sigma_{a}(T)$. Thus $\sigma_{w}(T) \backslash \sigma_{u w}(T) \subset \sigma(T) \backslash \sigma_{a}(T)$. Conversely, it easily seen that without condition on $T$, we have always that $\sigma(T) \backslash \sigma_{a}(T)=\sigma_{w}(T) \cap\left(\sigma_{a}(T)\right)^{C}$, and so $\sigma(T) \backslash \sigma_{a}(T) \subset \sigma_{w}(T) \backslash \sigma_{u w}(T)$. Hence, $\sigma_{w}(T) \backslash \sigma_{u w}(T)=\sigma(T) \backslash \sigma_{a}(T)$.

$\Longleftrightarrow$ ) Assume that $\sigma_{w}(T) \backslash \sigma_{u w}(T)=\sigma(T) \backslash \sigma_{a}(T)$, then $\sigma_{w}(T)=\sigma_{u w}(T) \bigsqcup\left(\sigma(T) \backslash \sigma_{a}(T)\right)$. Hence

$$
\begin{aligned}
\Delta_{a}(T) \cap \sigma_{w}(T) & =\Delta_{a}(T) \cap\left[\sigma_{u w}(T) \bigsqcup\left(\sigma(T) \backslash \sigma_{a}(T)\right)\right] \\
& =\left[\Delta_{a}(T) \cap \sigma_{u w}(T)\right] \bigsqcup\left[\Delta_{a}(T) \cap\left(\sigma(T) \backslash \sigma_{a}(T)\right)\right] \\
& =\left[\Delta_{a}(T) \cap\left(\sigma(T) \backslash \sigma_{a}(T)\right)\right] \subset \sigma_{a}(T) \cap\left(\sigma(T) \backslash \sigma_{a}(T)\right)=\emptyset .
\end{aligned}
$$

Theorem 3.12. Let $T \in L(X)$. Then the following statements are equivalent:

(i) $T$ satisfies property $\left(U W_{\Pi}\right)$ and $\Pi_{a}(T) \cap \sigma_{u w}(T)=\emptyset$;

(ii) $T$ satisfies property $\left(Z_{\Pi_{a}}\right)$ and $\sigma_{w}(T) \backslash \sigma_{u w}(T)=\sigma(T) \backslash \sigma_{a}(T)$;

(iii) $T$ satisfies property $\left(Z_{\Pi_{a}}\right)$ and $\Delta_{a}(T) \cap \sigma_{w}(T)=\emptyset$.

Proof. $($ ii $) \Longleftrightarrow($ iii $)$ Is an immediate consequence of Lemma 3.11

$(i) \Longrightarrow($ ii $)$ Follows directly from Theorem 3.7 and [5, Theorem 3.5].

$($ iii $) \Longrightarrow($ i $)$ Since $T$ satisfies property $\left(Z_{\Pi_{a}}\right)$ then $\Pi_{a}^{0}(T)=\Pi_{a}(T)$, see [8, Lemma 2.9]. As $\Pi_{a}^{0}(T) \cap \sigma_{u w}(T) \subset \Delta_{a}(T) \cap \sigma_{w}(T)$, then $\Pi_{a}(T) \cap \sigma_{u w}(T)=\emptyset$. Let us to prove that property $\left(U W_{\Pi}\right)$ holds for $T$. Since $\Delta_{a}(T) \cap \sigma_{w}(T)=\emptyset$, then $\Delta_{a}(T)=\Delta_{a}(T) \cap\left(\sigma_{w}(T)\right)^{C}=\Delta(T)$. As $T$ satisfies property $\left(Z_{\Pi_{a}}\right)$, then $\Delta_{a}(T)=\Pi(T)$.

Remark 3.13. If $T \in L(X)$ satisfies property $\left(Z_{\Pi_{a}}\right)$ and the a-Browder's theorem, then $T$ satisfies the property $\left(U W_{\Pi}\right)$. Indeed, since $T$ satisfies the property $\left(Z_{\Pi_{a}}\right)$, then $\Pi_{a}^{0}(T)=\Pi(T)$. a-Browder's theorem for $T$ entails that $\Pi_{a}^{0}(T)=\Delta_{a}(T)$. Hence $\Delta_{a}(T)=\Pi(T)$. So $T$ satisfies the property $\left(U W_{\Pi}\right)$. Remark that if $T$ satisfies property $\left(Z_{\Pi_{a}}\right)$ and $\Delta_{a}(T) \cap \sigma_{w}(T)=\emptyset$ then $T$ satisfies aBrowder's theorem.

Now, we give a similar result to Theorem 3.12 for the property $\left(Z_{E_{a}}\right)$.

Theorem 3.14. Let $T \in L(X)$. Then the following statements are equivalent:

(i) $T$ satisfies property $\left(U W_{E}\right)$ and $E_{a}(T) \cap \sigma_{u w}(T)=\emptyset$;

(ii) $T$ satisfies property $\left(Z_{E_{a}}\right)$ and $\sigma_{w}(T) \backslash \sigma_{u w}(T)=\sigma(T) \backslash \sigma_{a}(T)$;

(iii) $T$ satisfies property $\left(Z_{E_{a}}\right)$ and $\Delta_{a}(T) \cap \sigma_{w}(T)=\emptyset$.

Proof. $($ ii $) \Longleftrightarrow($ iii $)$ Is already done above.

(i) $\Longrightarrow($ ii $)$ Follows directly from Corollary 3.3 and Theorem 3.8

$($ iii $) \Longrightarrow\left(\right.$ i) Since $T$ satisfies property $\left(Z_{E_{a}}\right)$ then $T$ satisfies property $\left(Z_{\Pi_{a}}\right)$ and $E_{a}(T)=\Pi_{a}(T)$, see [8, Corollary 2.5]. It follows from Theorem 3.12, that $T$ satisfies property $\left(U W_{\Pi}\right)$ and $E_{a}(T) \cap$ $\sigma_{u w}(T)=\emptyset$, As $E(T)=\Pi(T)$ see [8, Lemma 2.3]. Then by Corollary 3.3, $T$ satisfies property $\left(U W_{E}\right)$ and $E_{a}(T) \cap \sigma_{u w}(T)=\emptyset$; 
Remark III: It is proved in [2, page 37] that if $T \in L(X)$ is a finite-isoloid operator, then $\sigma_{b}(T)=\sigma_{d}(T)$. Its proof is based on the fact that

$$
\text { if } \lambda \notin \sigma_{d}(T) \text {, then } \lambda \in \text { iso } \sigma(T) . \quad\left(I_{3}\right)
$$

But this is not true as we can see in the following example: we consider the right shift operator $R$ defined on $l^{2}$. We have $R$ is finite-isoloid and $\sigma_{d}(R)=D(0,1)$; but iso $\sigma(R)=\emptyset$. Note that the mistake $\left(I_{3}\right)$ is originated in $[3]$.

For $T \in L(X)$, we denote by $E_{f}(T)=\{\lambda \in$ iso $\sigma(T): \alpha(T-\lambda I)<\infty\}$, and we say that $T$ is finitely if iso $\sigma(T)=E_{f}(T)$.

Now we give in the next proposition, the same version followed by a correct proof and under the weaker hypothesis that $T$ is finitely. We observe that if $T$ is finite-isoloid, then $T$ is finitely. Observe also that $\sigma_{b}(T)=\sigma_{d}(T) \Longleftrightarrow \Pi(T)=\Pi^{0}(T)$.

Proposition 3.15. If $T \in L(X)$ is a finitely operator, then $\sigma_{b}(T)=\sigma_{d}(T)$.

Proof. The inclusion $\sigma_{d}(T) \subset \sigma_{b}(T)$ is always true. Let $\lambda \notin \sigma_{d}(T)$ be arbitrary. If $\lambda \notin \sigma(T)$ then $\lambda \notin \sigma_{b}(T)$. If $\lambda \in \sigma(T)$, then $\lambda \in \Pi(T)$ and so $\lambda \in$ iso $\sigma(T)$. As $T$ is finitely then $\lambda \in \Pi^{0}(T)$ and $\lambda \notin \sigma_{b}(T)$. Hence $\sigma_{b}(T)=\sigma_{d}(T)$.

For giving the reader a good overview of the subject, we give another very simple proof. Since $T$ is finitely, then $\Pi^{0}(T)=\Pi(T) \cap E_{f}(T)=\Pi(T)$.

Remark IV: In the paper [7, the authors defined a property named $(B v)$ as follows: $T \in L(X)$ satisfies property $(B v)$ if $\sigma(T) \backslash \sigma_{u w}(T)=\sigma(T) \backslash \sigma_{u b}(T)$. Moreover, they studied this property in connection with different known Weyl type theorems and properties. But it is trivially that this property, is equivalent to the classical a-Browder's theorem; and all of the results announced in this paper are already done.

\section{References}

[1] P. Aiena, Fredholm and Local Spectral Theory II, with Application to Weyl-type Theorems, Springer Lecture Notes of Math no. 2235, (2019).

[2] P.Aiena, M. Kachad, Property $\left(U W_{\Pi}\right)$ and perturbations, Annals of Functional Analysis, 11 (2020), 29-46.

[3] P.Aiena, M. Kachad, Property $\left(U W_{\Pi}\right)$ and localized SVEP, Acta Sci. Math., 84 (2020), 555571.

[4] M. Berkani, M. Kachad, New Weyl-type theorems -I, Func. Anal. Approx. Comp., 4 (2012), $41-47$.

[5] M. Berkani, M. Kachad, New Browder and Weyl type theorems, Bull. Korean Math. Soc., 52 (2015), 439-452. 
[6] M. Berkani, H. Zariouh, Extended Weyl type theorems, Math. Bohemica 134 (2009), 369-378.

[7] N. Jayanthi, P. Vasanthakumar, A new Browder type property, Inter. J. Math. Anal., 14 (2020), 1-11.

[8] On the property $\left(Z_{E_{a}}\right)$, Rend. Circ. Mat. Palermo, 65 (2016), 323-331.

Zakariae Aznay,

Laboratory (L.A.N.O), Department of Mathematics,

Faculty of Science, Mohammed I University,

Oujda 60000 Morocco.

aznayweyl@gmail.com

Hassan Zariouh,

Department of Mathematics (CRMEFO),

and laboratory (L.A.N.O), Faculty of Science,

Mohammed I University, Oujda 60000 Morocco.

h.zariouh@yahoo.fr 